\title{
New Approach for Address Auto-Configuration in MANET Based on Virtual Address Space Mapping (VASM)
}

\author{
Majid Taghiloo ${ }^{1}$, Mehdi Dehghan ${ }^{2}$, Jamshid Taghiloo ${ }^{3}$, Maria Fazio ${ }^{4}$, \\ ${ }^{1}$ Computer Engineering Department, Amirkabir University of Technology, Tehran, Iran \\ ${ }^{2}$ Computer Engineering department, Amirkabir University of Technology, Tehran, Iran \\ ${ }^{3}$ Computer Engineering Department, Iran University of Science and Technology, Tehran, Iran \\ ${ }^{4}$ Mathematic Department, Messina University, Messina, Italy
}

\begin{abstract}
Mobile Ad Hoc Networks (MANETs) are networks with self-organizing capabilities and without a fixed infrastructure. Wireless nodes communicate among themselves using multi-hop radio relaying, without requiring the packets to pass through a central access point or a base station. Routing is a function of speed and stability with which the nodes are able to acquire addresses. Efficient routing forms the basis of a fast and reliable communication network. In a highly mobile and infrastructure-less scenario, pre-configuration of addresses is not possible. Therefore node addresses need to be configured dynamically with minimum delay and packet loss. The main task of an address auto-configuration protocol is to manage the resource address space. It must be able to select, allocate, and assign a unique network address to an un-configured node. This paper proposes a new address auto-configuration protocol for mobile ad hoc networks. The scheme uses virtual address space for addressing new nodes joining a network. The aim is to map one point from virtual address sheet to exactly one new node. The reason for using the term "virtual" is that the whole corresponding address space is a 2D flat sheet and each point of this sheet is virtually mapped to a node in MANET. The protocol uses coordinate values for generating addresses.
\end{abstract}

Keywords: Ad Hoc Network, MANET, Auto-Configuration, IP Address,

\section{INTRODUCTION}

A Mobile Ad Hoc Network (MANET) consists of a set of homogenous mobile nodes communicating with each other via wireless links, which cooperatively and spontaneously form an IP-based network. This network is independent of any fixed infrastructure or centralized administration. A node communicates directly with nodes within its wireless communication range. Nodes that are part of the MANET but beyond each other's wireless range, communicate using a multi-hop route through other nodes in the network. The network's topology may change rapidly and unpredictably. Such a network may operate in a stand-alone fashion, or may be connected to the larger Internet.

The major research efforts in the area of mobile ad hoc networks (MANET) focus on developing efficient routing protocols assuming that IP addresses of mobile hosts have been already configured before joining the network. Providing IP address auto-configuration mechanism for MANET is still an open problem.

Dynamic configuration in a wired network is accomplished using Dynamic Host Configuration Protocol (DHCP). However, it requires the presence of a centralized DHCP server which maintains the configuration information of all nodes in the network. Since a MANET is devoid of any fixed infrastructure or centralized administration, this approach cannot be used.

Since in a highly mobile, infrastructure-less scenario, preconfiguration of addresses is not possible, the node addresses need to be configured dynamically with minimum delay and packet loss. The main task of an address auto-configuration protocol is to manage the resource address space. It must be able to select, allocate, and assign a unique network address to each new node. When a node leaves the network, the corresponding address must eventually be de-allocated to prevent exhaustion of the address space. The proposed aims at mapping one point from virtual address sheet to exactly one new node using a virtual address space. The reason for using "virtual" word in this article is that the whole corresponding address space is 2D flat sheet and each point of this sheet is virtually mapped to one node in MANET. Every address autoconfiguration protocol should fulfill the following requirements:

- Assign unique address for each node in network.

- Use the entire address space in address allocation process.

- Solve the problems associated with merging and partitioning of networks

\section{RELATED WORKS}

We can classify all address auto-configuration protocols into two categories: statefull and stateless.

\section{A. Stateful Approaches}

Auto-configuration protocols for Ad hoc networks following a stateful approach can be classified according to the 
way they maintain the address allocation table. Three major approaches are [1]:

- Centralized maintenance of the allocation table [2]

- Distributed maintenance of a common allocation table [3]

- Distributed maintenance of multiple disjoint allocation tables [4]

With a centralized allocation table, only one node in the network is allowed to select addresses for un-configured nodes. Hence this node must be permanently reachable. To achieve this, the node representing the central entity is usually selected dynamically, and thus may change. Because transferring the allocation table from the old entity to a new one is not always possible, the allocation table of the new entity may be empty. Hence all nodes have to be notified reliably for either reregistering or acquiring new addresses. The latter results in a number of unnecessary address changes. Although a backup entity might alleviate this problem, both alternatives are problematic in highly dynamic scenarios where the central entity changes frequently. Furthermore, the central entity may represent a bottleneck in large networks.

In contrast, a distributed allocation table allows every node to select addresses for un-configured nodes, but synchronization between all nodes is necessary to avoid the assignment of duplicate addresses. With common allocation table consistency, in case of multiple disjoint tables, the disjointedness of the tables must be guaranteed. Efficient and reliable synchronization is the major challenge of this approach. It may consume a considerable amount of bandwidth since this usually requires reliable or periodic flooding. Either case, inconsistencies may result in unresolved address conflicts or address leaks. Network merging must be handled specifically to synchronize the allocation tables of the participating networks and to identify address conflicts after the merge.

\section{B. Stateless Approaches}

Stateless approaches do not maintain allocation tables. Instead, an un-configured node assigns an address to itself which is generated randomly or is based on hardware ID. The uniqueness of the address is verified by a DAD procedure. Hence, the DAD is the most important part of these protocols. Address configuration can be done either before or after completion of the DAD. The former is also called optimistic DAD.

Here, the problem again is to support network merging: DAD can be either repeated after network merging or performed routinely. For the former, the network merging must be detected and all nodes reliably notified in order to repeat the DAD procedure. This usually requires a reliable flooding. Moreover, concurrent DADing of all nodes may result in a broadcast storm. A permanent DAD can either be realized by periodic execution or by integration with the routing protocol. The latter is usually more efficient in terms of protocol overhead and can prevent misrouting of data packets due to conflicts. However, it may take more time to detect conflicts.
In case of reactive routing protocols, only conflicts between active nodes can be detected.

\section{DEFINITIONS}

In this protocol, nodes are classified into four categories:

- Allocator: Maintain the address space. They allocate new addresses for joining nodes.

- Initiator: An intermediate node between Allocators and Requester node that exchange all messages between them.

- $\quad$ Requester: new node that needs to get IP address in order to join the network.

- Normal: all other nodes are in this category.

Each Allocator in the network contains a disjoint address space. Therefore, address space overlap between Allocators is none.

\section{BASIC IDEA}

According to this protocol, when a new node goes into adhoc mode, it sends a single-hop INITIATOR_SEARCH message, in order to find an Initiator. If there is no reply for this packet, the node assumes that it is the only node in that network and starts the network setup process. If the joining node gets more than one response, it selects the sender of the first arrived packet as Initiator and sends him an address request packet. As shown in Figure 1, the main task of Initiator is to obtain a new IP address from its Allocator and assign it to the requesting node (Requester).

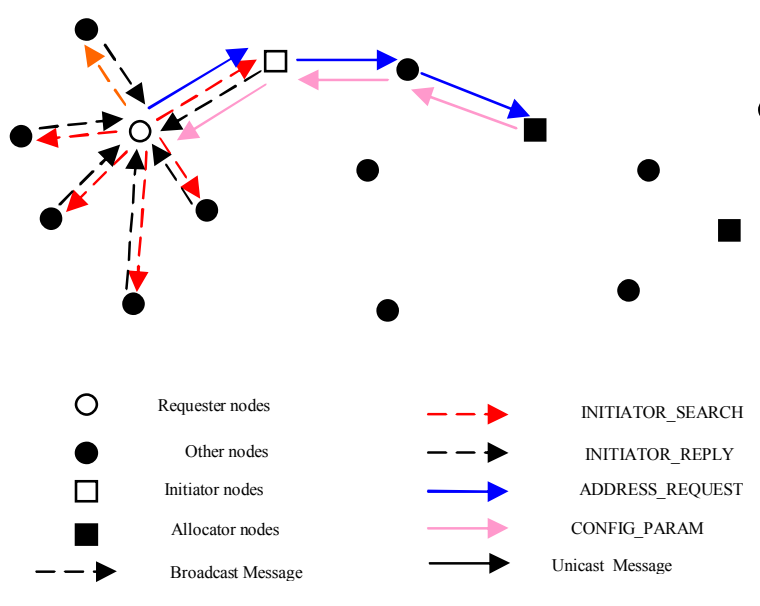

Figure 1. Address allocation for new node

If the received response was from an Allocator, select this node as its Allocator and asks for a new address immediately.

As mentioned before, in this protocol, each network has at least one Allocator. Each Allocator contains an address space used for assigning unique IP addresses for the newly joined nodes. Method of choosing nodes as Allocator and the way address space is assigned for that node is the chief task of this protocol. In addition to generating unique IP addresses, an Allocator can create another Allocator in the network for 
balancing the overhead of protocol traffic and minimizing the time of address assignment for new nodes. For efficient management of network merging and partitioning process, each Allocator holds a list of all Allocators in the network. The number of Allocators in each network is limited. Thus the size of Allocators' list will be very small.

The main difference between this protocol and other stateful protocols is in its bottom-up approach for address space distribution.

The protocol benefits from the following timers:

- tJoin: Requester node sets this timer after sending INITIATOR SEARCH message and stops this timer after receiving INITIATOR_REPLY packet.

- tAlloc: The Initiator node activates this timer after sending ADDRESS_REQUEST packet to its own Allocator and stops that after receiving CONFIG_PARAM packet.

- tInit: By using this timer, the protocol can manages Initiator failure or departure.

- tAllocatorSync: This timer is used to synchronizing Allocator's table.

\section{ALLOCATOR SELECTION MECHANISM}

Address space corresponding to the Allocator is a square in the main address space sheet and its side length is specified by SQUARE_SIDE. Figure 3 shows the method of address space expansion. Each square is surrounded by eight other squares. For convenience, each square is assigned a number from one to eight (Figure 3-b). Each Allocator node, based on its current state value, can create one, three, or eight Allocators. Thus, Allocators corresponding to the squares one, three, five, and seven, can add one Allocator, and Allocators corresponding to the squares two, eight, four, and six, can add three Allocators. Finally, the Allocator corresponding to node zero, can add eight Allocators to the network. New Allocator creation is directly related to the method of address space expansion. This protocol, for structuralizing of this process, proposes address space propagation pattern based of the current state of node (parent Allocator).

ALLOC_BYTE is an eight bits data structure; each bit represents one of the surrounding squares. Each bit number is equal to its corresponding square number. As shown in Figure 3.a, for each state from 0 to 9 , there is a value stored in ALLOC_BYTE. Number of zero values represents the maximum number of Allocators that can be created by this one.

The values of ALLOC_BYTE and ALLOC_NUM (embedded in four least significant bits of ALLOC_STATE) are dedicated to Allocator creation. ALLOC_NUM shows the amount of Allocators created by the node. If the value of ALLOC_NUM is equal to the number of Zero bit in ALLOC_BYTE, it denotes that this node has no grant for creating new Allocator. While creating new Allocator, protocol increments the content of ALLOC_NUM by one.
Figures 3.b, 3.c, and 3.d show the ordered expansion of address space. Direction of arrows displays the address expansion's direction. For example, imagine the address space for a node with state 1 in figure 3.c. With due attention to Figure 3.a, only first bit is zero in the value of ALLOC_BYTE. It means that the address space can only be expanded in the arrow direction corresponding to the square 1 in Figure 3.c. Hence, the Allocator sends state 1 to the new Allocator. Figure 3.d shows the method for address space expansion. Different numbers show the change in state and expansion of address space for the Allocator corresponding to each square.

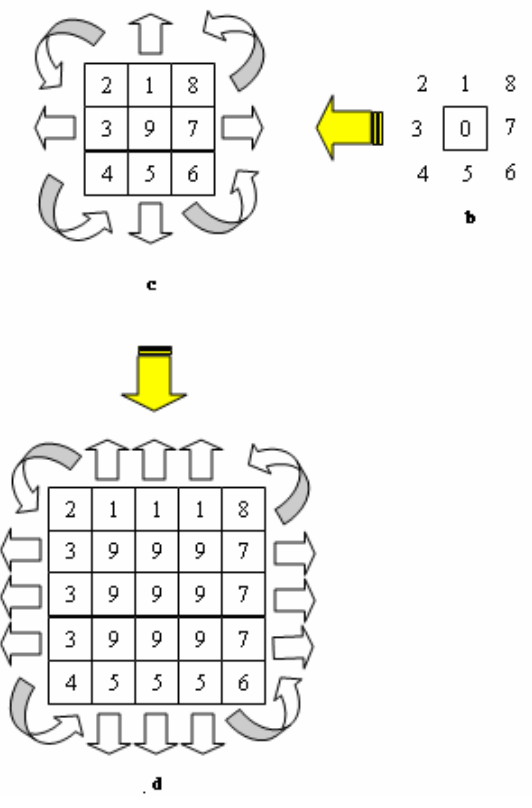

\begin{tabular}{|c|c|}
\hline $\begin{array}{c}\text { Alloc_Byte } \\
\text { Value }\end{array}$ & $\begin{array}{c}\text { Allocator } \\
\text { State }\end{array}$ \\
\hline 00000000 & 0 \\
\hline 11111110 & 1 \\
\hline 11111000 & 2 \\
\hline 11111011 & 3 \\
\hline 11100011 & 4 \\
\hline 11101111 & 5 \\
\hline 10001111 & 6 \\
\hline 11011111 & 7 \\
\hline 00111110 & 8 \\
\hline 11111111 & 9 \\
\hline \multicolumn{2}{|c}{$\mathbf{a}$} \\
\hline
\end{tabular}

Figure 2. Address space expansion method

When an Allocator creates another one, it should initialize the states (ALLOC_BYTE) of that new Allocator. Figure 4 depicts the state diagram for this process. Each rectangle represents an Allocator. Inside each Allocator, there are two numbers separated by '/' (except the first Allocator which is the root). The left number denotes the current state of the parent Allocator when a child Allocator is created. For example the root has created eight children. Upon creating the eight child, the root changes its state to 9 , which means it will not be able created any more children. The right number is the initial state of the -to be created- children. This process will go on until the boundary of the address space is touched and no more Allocator can be created.

\section{PROTOCOL DETAIL}

In this section, protocol operations are discussed. The methods of dealing with ad hoc network specific challenges are described here:

\section{A. Network Initialization}

Initially, the very first node starts sending a single-hop INITIATOR_SEARCH message. The Requester waits for tJoin 
timer to timeout. As there is no other node in the network, the Requester will not receive any reply. After the timeout, the requesting node retries sending joining packets up to JOINING PKT RETRY times. This constant is the threshold value of the sending joining packets. This is to ensure that, if there are other configured nodes in the network, in the event of message losses, the node does not assume itself as the first network node. When all the retries failed, the node concludes that it is the only node in the network. At this stage, network initialization setup should start.

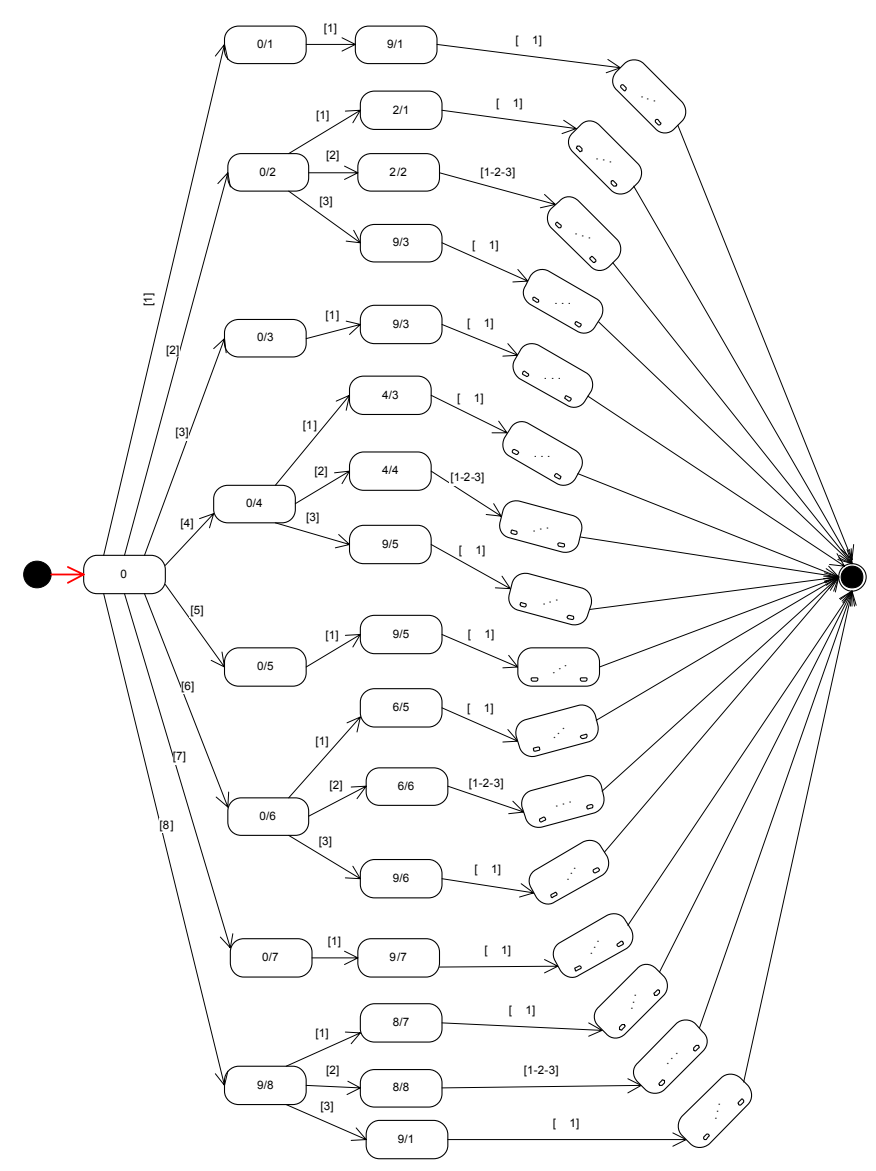

Figure 3. State Diagram of Allocators

Note that, this approach assumes the address space as a latticed square sheet filled by squares with side SQUARE_SIDE.

The node then selects random square from address space sheet. Then, it initializes its state (ALLOC BYTE) by 00000000 and sets the value of ALLOC_NUM, to zero. The number of zeros in ALLOC BYTE indicates that this node can create eight Allocators. Finally, it selects randomly STEP value from $(1,8)$ and NetID. It uses coordination of STEEPth point from bottom-left corner of square as its IP address.

STEP specifies the start point of address allocation. For next address assignment, Allocator should add eight to the last allocated point. Point selection sequence is from bottom left corner to the top right corner. The main objective of using STEP is to decrease the probability of address conflict after networks merging. Furthermore, this parameter can be useful in reducing the traffic overhead of managing duplicate addresses.

\section{B. Joining new node}

After finishing of the network initialization process, when a new node appears near a configured node, the new node sends INITIATOR_PACKET packet as a one hop broadcast message with source address Temp_ID. Then it waits for tJoin units of time. If the receiver of INITIATOR packet is an Allocator, it can introduce itself as an Initiator, allocate new IP address and send configuration parameter to the requester directly.

Otherwise, if the receiver is not an Allocator, that node changes its state to Initiator and then send address request packet (ADDRESS_REQUEST) to its Allocator and waits for tAlloc units of time. Configuration parameter contains a new allocated IP address and IP address of Allocator. As Initiator is intermediate, it sends address request packet to its Allocator. And then waits for tAlloc units of time. Upon receiving ADDRESS REQUEST packet, the Allocator allocates new address and then sends it in the form of CONFIG_PARAM packet to Requester node. If the sender of ADDRESS_REQUESTER packet is an Initiator, it should send this configuration packet to the Requester.

\section{New address allocation in Allocators}

Address space of each Allocator maps to a square in the address sheet. Hence, every Allocator in the network has a mutually exclusive set of addresses. Address allocation process should be done in a way that minimizes the address conflict and also prevents wasting of address space.

The advantage of the proposed protocol, unlike [3], is that its Allocators hold some property of their corresponding square. The properties include coordination of bottom left corner, and last allocated point.

The simplest way to allocating a new address is selecting points from bottom left corner of the square to top right corner. But the probability of selecting the same square is higher than selecting the same point in the address sheet. The protocol has devised a method for solving this problem. For this purpose, STEP variable is used by setting its bits to one in a randomly fashion. The benefit of this method is that it decreases the probability of address conflict during network merging as the same square from two Allocators in separate networks may have been be assigned. Initially, all 8 bits in STEP variable are 0 . A random number from 1 to 8 is selected and the corresponding bit in STEP variable is sets to 1 .

The resulting value of STEP is assigned to CURRENT STEP. At the next iteration, another bit from the 7 remaining bits is set to 1 in order to assign a new value to CURRENT_STEP. This process will continue until all 8 bits of STEP are set to 1. For address allocation, Allocator first starts from the CURRENT_STEPth point in its square and then, it adds eight to the last allocated point for allocating the next address. If the coordination of the new point is out of range, Allocator will continue with the next iteration to generate 
another STEP value. CURRENT STEP is embedded in four most significant bits of ALLOC_STATE. If the FREE_ADDR_LIST is not empty, it is better to use IP address of depart nodes. It helps solving address exhaustion problem.

\section{Allocators Synchronization}

For speedy updating Allocator's tables, the protocol needs a synchronization mechanism between them. This process is useful for abruptly departure of Allocators.

For doing this, Allocators should announce their state changes to other Allocators periodically using UPDATE_ALLOC_TABLE packet. It uses tAllocatorSync timer for scheduling. If a significant change was occurred in Allocator tables, it is possible to send more packets regardless of due periods.

\section{E. departure of nodes}

The nodes in the network can either depart abruptly or gracefully from the network.

\section{1) Graceful departure}

The protocol assumes two modes for Allocator nodes: Normal Mode (NM) and Resource Saving Mode (RSM).

$\mathrm{FD}=$ Set of all depart node's address

$\mathrm{S}=$ All address space of Allocator

$\mathrm{A}=$ Set of all allocated addresses

$\operatorname{MAX}(\mathrm{A})=$ Last allocated address

Len $(A)=$ Number of elements in set $A$

$F_{D}=\{\forall x \mid x \in S \wedge x \notin A \wedge x \leq<A X(A)\}$

If $\frac{\operatorname{Len}(A)}{2}<\operatorname{Len}\left(F_{D}\right)$ and mode $=\mathrm{NM}$ then

Set mode $=$ RSM

$$
\text { Else if } \begin{gathered}
\frac{\operatorname{Len}(A)}{2} \geq \operatorname{Len}\left(F_{D}\right) \\
\text { Set mode }=\mathrm{NM}
\end{gathered} \text { and mode }=\mathrm{RSM} \text { then }
$$

The above formulas states when an Allocator switches between the two modes.

Every Allocator node uses dynamic list (FREE_ADDR_LIST) for reclaiming IP address of depart nodes. In the worst case, the maximum size of the list is half the number of allocated addresses for each Allocator. This was the case in NM.

A node wishing a graceful departure sends a departure request to one of its neighbors before leaving the network. If this node is an Allocator, it sends DEPART_ALLOCATOR message giving coordination of bottom left corner of its square, last allocated point, ALLOC BYTE, ALLOC STATE, STEP, FREE_ADDR_LIST and Allocator tables, to one of its neighbors. There are several methods for selecting this neighbor. An example can be invoking the hello message of the routing protocol. Also, the selected neighbor being a normal node is the first priority. If the leaving node receives more than one message from its neighbors, it can select a node with greater IP address. After receiving departure message, the neighbor node changes its own address and state to the leaving Allocator's address and state. The new Allocator also sends DEPART REGULAR message to its parent Allocator. The parent adds the IP address of that normal node to FREE_ADDR_LIST.

One exception can occur upon departure which is when that all neighboring nodes are Allocators. In this situation, the departing Allocator will select one of its child normal nodes and then, sends its current state to that node. Furthermore, it may happen that all normal nodes of the Allocator departed from network. It is the time that the departing Allocator hands the task for finding a replacement for itself to one of its child Allocators. In the worst case, if none of the children could help their parent, the Allocator removing process will start.

When the depart node is normal, it sends the DEPART_REGULAR packet to its Allocator. If the receiving Allocator is in NM mode, it adds the node's IP address to FREE ADDR LIST and increments FREE_ADDR_LIST_LEN by one. In case of RSM, the only task of Allocator is incrementing FREE_ADDR_LIST_LEN.

As mentioned before, when the neighbor of the departing Allocator is an Allocator, it suffices to relaying the DEPART ALLOCATOR packet. So, if the parent Allocator of departs node is in the network, it can see this message. Then it starts tDepart timer and waits for receiving DEPART REGULAR packet from depart node. After tDepart timeout, if there is no DEPART REGULAR packet received, the parent Allocator supposes that there is no normal child node for the departing Allocator.

\section{2) Abrupt departure of nodes}

The assumption that every node leaves the network after broadcasting depart message is not correct. Some nodes may crash or switch off without prior notice and some other may go out of range. Consider a network in which some Allocators have crashed. The network doses not have any address remaining, except the crashed address pool. The address loss would be detected as soon as a new node joins the network.

If no free IP addresses are found during the address allocation, it dose not necessarily mean that there are no free IP addresses in the network. It is possible that some nodes may have left the network abruptly. Hence, reclamation of addresses needs to be done whenever the nodes have left the network abruptly. This feature solves the scalability problem of this protocol. Protocol simply searches all of its child nodes, and then it can assign absent nodes addresses for new nodes. It should be mentioned that, if the abruptly leaving node is an Allocator, the synchronization process which is performed earlier, can detect and replace the absent Allocator.

\section{F. Requester movement}

Imagine a requester asks an initiator for an address and moves to a second Initiator before the first Allocator could assign an IP address to the Requester. The Requester will give the new Initiator the IP address of the old Allocator. New 
Initiator then sends IP Address Forward message to the old Initiator. Upon receiving the IP Address Forward message, Old Initiator updates the entry for the corresponding requester with the IP Address of the new Initiator. The same applies to Allocators when requester has direct connection to Allocator.

Alternately, the old Allocator could abort the IP address Allocation process when the requester migrates and the new Allocator could start the allocation process again. In the former approach, the communication overhead is reduced by maintaining state information. The latter does not maintain such state information but requires the address allocation process to be started all over again.

\section{G. Initiator failure}

There is a possibility that the Allocator (or Initiator) node crashes. This situation is handled by tjoin (for initiator) and tsearch (for Allocator) timers. After sending the request message, Requester (or Initiator) waits to hear a reply tJoin (or tSearch) unit of time. After the time out, the Requester (or Initiator) will retry broadcasting the Request message for a certain number of times (threshold). After meeting the threshold the process will repeat for finding another initiator.

\section{H. Packet loss}

As mentioned before, it may happen that any of the protocol messages may get lost. Timers and threshold values are considered to handle all message loss cases.

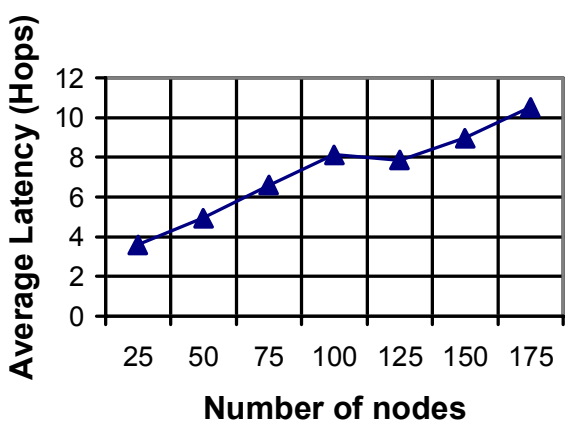

Figure 4. VASM Auto-Configuration average latency in resolving address

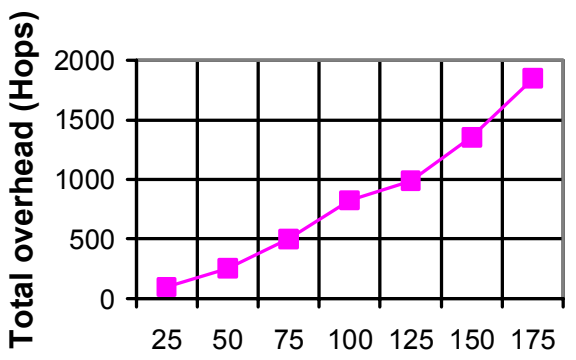

Number of nodes
Figure 5. VASM Auto-Configuration traffic overhead

\section{SIMULATION EXPERIMENTS}

Simulation experiments were performed using the behavioral analyzing to evaluate the performance of the protocol in terms of overhead and latency. The random waypoint mobility model was used. The speed of nodes in the network was selected randomly between 1 to 15 meters per second and the pause time was 10 seconds. The simulation duration was 3600 seconds. The JOINING PKT RETR threshold was 2, and the SQUARE_SIDE and SHEET SIDE were set to $0 \times 8$ and $0 x F F F F$ respectively. The network area was a square of $670 \mathrm{~m} \times 670 \mathrm{~m}$. Transmission range of the nodes selected randomly from 150 to 300 .

Using time parameters for protocol analyzing purpose is not precise metric, because it depends on the environment configuration of simulator and simulation scripts. In each autoconfiguration protocol, the number of hops that packets traverse in network can be very useful and realistic parameter for performance analyzing purpose if the mobility model and link breakage (packet loss) be correctly added to the simulation. In this experiment, we have used this parameter to show performance of the protocol. Figure 4 shows the average latency of address resolving for different number of nodes. it denotes that the growing of nodes number has not salient affect into the latency. In figure 5, the overall traffic overhead of the protocol has been demonstrated. Number of nodes has direct relation with the number of Allocator synchronization packets that are multicast periodically.

\section{CONCLUSION}

We presented a distributed protocol for dynamic configuration of nodes in MANETs. We have addressed the issue of unique IP address assignments to nodes in the absence of any static configuration or central servers. The basic idea is to dynamically distribute virtual address space among the dynamically selected Allocator nodes. The management of virtual address space conflicts is simple because each Allocator node holds the topology of squares corresponding to the other Allocator nodes. Currently we are working on the Integration of virtual address space after merging of different networks.

\section{REFERENCES}

[1] K. Weniger, M. Zitterbart, "Address Autoconfiguration in Mobile Ad Hoc Networks: Current Approaches and Future Directions," IEEE Network Magazine Special issue on Ad Hoc Networking, July 2004.

[2] M. Gunes, J. Reibel, "An IP Address Configuration Algorithm for Zeroconf Mobile Multihop Ad Hoc Nrtworks," Proc. Int'l.Wksp. Broadband Wireless Ad Hoc Networks and Services, Sophia Anipolis, France, September 2002.

[3] S. Nesargi, R. Prakash, "MANETconf: Configuration of Hosts in a Mobile Ad Hoc Network." Proc. IEEE INFOCOM 2002, New York, NY, June 2002.

[4] M. Mohsin, R. Prokash, "IP Address Assignment in a Mobile Ad Hoc Network," Proc. IEEE MILCOM 2002, Anaheim, CA, October 2002. 